\title{
How Should We Measure Peripheral Spondyloarthritis?
}

\author{
Laura C. Coates ${ }^{1}(\mathbb{D})$ and William Tillett ${ }^{2}$ (ID
}
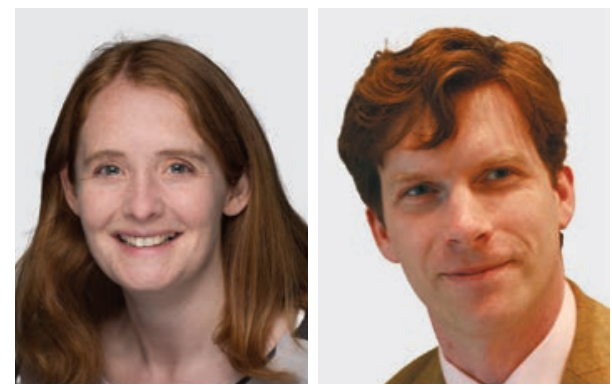

Spondyloarthritis $(\mathrm{SpA})$ is recognized as an overarching spectrum of disease characterized by axial SpA (axSpA), peripheral arthritis, enthesitis, and dactylitis. Despite significant overlap, patients are often characterized as having predominantly peripheral or axial involvement. There are advantages to the separate assessment of peripheral and axial disease, particularly in the randomized clinical trial (RCT) setting, in trying to understand the differential effectiveness of novel biologic and targeted synthetic drugs on the peripheral and axial skeleton. While some diagnoses within the $\mathrm{SpA}$ concept have attracted increasing research over recent decades, it seems that peripheral SpA ( $\mathrm{pSpA}$; excluding psoriatic arthritis $[\mathrm{PsA}]$ ) still lags behind.

Peripheral SpA, as defined by the Assessment of Spondyloarthritis international Society (ASAS) criteria, allows a diagnosis in patients with peripheral arthritis alongside other features of SpA. ${ }^{1}$ This encompasses PsA but also inflammatory bowel disease-related arthritis, reactive arthritis, and undifferentiated $\mathrm{pSpA}$, representing a diverse group of patients. To date, there have been large numbers of observational and treatment trials in PsA, but there are relatively few in the other forms of pSpA.

LCC is a National Institute for Health Research (NIHR) Clinician Scientist and Senior Clinical Research Fellow funded by an NIHR Clinician Scientist award. The research was supported by the NIHR Oxford Biomedical Research Centre. The views expressed are those of the authors and not necessarily those of the NHS, the NIHR, or the Department of Health.

${ }^{1}$ L.C. Coates, MBChB, PhD, Nuffield Department of Orthopaedics, Rheumatology and Musculoskeletal Sciences, University of Oxford, Oxford; ${ }^{2} W$. Tillett, MBChB, BSc, PhD, Royal National Hospital for Rheumatic Diseases, and Department of Pharmacy and Pharmacology, University of Bath, Bath, UK.

$W T$ has received research funding, consulting or speaker fees from AbbVie, Amgen, Celgene, Eli Lilly, Janssen, MSD, Novartis, Pfizer, and UCB. LCC has received grants/research support from AbbVie, Amgen, Celgene, Eli Lilly, Novartis and Pfizer; worked as a paid consultant for AbbVie, Amgen, Boehringer Ingelheim, BMS, Celgene, Eli Lilly, Gilead, Galapagos, Janssen, Novartis, Pfizer, and UCB; and has been paid as a speaker for AbbVie, Amgen, Biogen, Celgene, Eli Lilly, Galapagos, Gilead, Janssen, Medac, Novartis, Pfizer, and UCB.

Address correspondence to Prof. L.C. Coates, Botnar Research Centre, Windmill Road, Oxford OX3 7LD, UK.

Email:laura.coates@ndorms.ox.ac.uk.
The aim of the publication of these classification criteria was to support future research into this group.

However, in addition to classifying the condition, valid outcome measures are required to examine disease activity, response, and impact in $\mathrm{pSpA}$ in the $\mathrm{RCT}$ and clinical practice settings. The only large RCT of treatment for nonpsoriatic $\mathrm{pSpA}$ to date has been the ABILITY-2 study, which compared adalimumab (ADA) vs placebo. ${ }^{2}$ This study used the ASAS criteria for inclusion but also excluded patients with predominant axial disease or PsA, as studies of ADA in these populations had already been completed.

In this study, ${ }^{2}$ due to a lack of validated outcome measures addressing arthritis, enthesitis, and dactylitis in this population, a novel composite efficacy outcome measure was designed: the Peripheral SpA Response Criteria (PSpARC40). The PSpARC40 is defined as $\geq 40 \%$ improvement $(\geq 20$-mm absolute improvement) in the patient global and pain visual analog scale scores, and $\geq 40 \%$ improvement in one of the following: (1) peripheral joint count; (2) total enthesitis count; or (3) dactylitis count. ${ }^{2}$ While this study met its primary outcome of PSpARC 40 at Week 12 for ADA compared to placebo, no other validation data are published on this outcome. Interestingly, the trial cohort has attracted attention from researchers as there are few large studies in nonpsoriatic $\mathrm{pSpA}$, and subsequent papers have looked at the validation of "borrowed" outcome measures including the Bath Ankylosing Spondylitis Disease Activity Index (BASDAI), Ankylosing Spondylitis Disease Activity Score (ASDAS), American College of Rheumatology outcomes, and minimal disease activity as used in PsA. ${ }^{3,4}$ However, trial cohorts are never reflective of the more heterogenous real-world populations and so further research in additional real-world datasets in $\mathrm{pSpA}$ are needed.

The study by Beckers et $\mathrm{al}^{5}$ in this edition of The Journal of Rheumatology analyzed 3 different composite measures in $\mathrm{pSpA}$, with a focus on examining concurrent validity in the clinical practice setting. This represents a step forward toward validation of outcome measures in $\mathrm{pSpA}$ that could support future research, and is strengthened with the use of data from a real-world dataset.

\section{See Peripheral SpA measures, page $x x x$}


The study used data from a Dutch SpA registry and focused on 304 patients with $\mathrm{pSpA}$ having treatment in routine clinical practice. The study included data on the Disease Activity in PsA (DAPSA), PsA Disease Activity Score (PASDAS), and ASDAS. All these measures correlated well with individual measures of disease activity or impact such as patient/physician global scores and quality of life measures. The ASDAS performed well despite the focus on peripheral SpA rather than axial symptoms, although this is in keeping with previous studies of BASDAI and ASDAS in PsA populations. ${ }^{67}$ The DAPSA and PASDAS, which were designed for PsA, also performed well, although the proportion of patients classified with active disease activity (moderate-high disease) by the DAPSA was a lot lower than the other measures. This may be because the score is proportional to the tender and swollen joint counts, which may not accurately reflect disease in a predominantly oligoarticular disease.

Unfortunately, as this study ${ }^{5}$ was reliant on real-world data collection within the clinic, quite a high number of patients had missing data, particularly for the DAPSA and PASDAS. The other key limitation is that because most patients with $\mathrm{pSpA}$ have PsA, this study includes a limited number of patients with nonpsoriatic pSpA (82 of 304 patients), where data are particularly needed.

One of the widely recognized challenges in the pSpA field is the heterogeneous nature of disease. For example, $\mathrm{pSpA}$ could encompass a patient with PsA and asymptomatic axial disease, and a patient with nonpsoriatic $\mathrm{pSpA}$ monoarthritis. The DAPSA and PASDAS were developed for the purpose of assessing peripheral joint involvement in PsA, whereas the ASDAS was developed to assess disease activity in axSpA. As Beckers et al highlight, a composite measure that includes psoriasis $(\mathrm{PsO})$ may not be appropriate in a patient without $\mathrm{PsO}_{s}{ }^{5}$ The same concern could apply to those with PsA and no axial involvement where instruments such as ASDAS may not be appropriate. In both instances, the instrument may lack face validity. A further challenge is the influence of axSpA and $\mathrm{pSpA}$ on an instrument and for this reason, subanalyses are often made on modified versions of the BASDAI and ASDAS to try and separate out change in axial and peripheral symptoms. The question that arises is whether we should be repurposing outcome measures developed for subtypes of axSpA to apply to the axSpA population as a whole, or whether some subtypes are sufficiently different and prevalent to justify dedicated outcome measures.

The same dilemma of lumping or splitting exists with respect to axial involvement in PsA, where there the exact nature of axial involvement in PsA is yet to be determined. To that end, a collaboration between the Group for Research and Assessment of Psoriasis and Psoriatic Arthritis (GRAPPA) and ASAS has been formed to study axial PsA in a multinational, multicenter cross-sectional study. The Axial Involvement in Psoriatic Arthritis Cohort (AXIS) study aims to enroll 400 patients to determine the prevalence and phenotype of axial PsA using imaging, clinical, and laboratory assessments. ${ }^{8}$ The collaboration between ASAS and GRAPPA in the AXIS study could be a future forum to validate or develop a novel composite instrument for use across the spectrum of $\mathrm{pSpA}$.
The Outcome Measures in Rheumatology (OMERACT) method for selecting an assessment can be helpful when considering the relative merits of an assessment tool. The instrument selection process starts with understanding what you are trying to measure, including the domains of disease and context such as RCTs, observational studies, or clinical practice, where the requirements of an instrument will vary. The next steps OMERACT recommends are identification of candidate instruments, determining domain match and feasibility, narrowing the field, and synthesizing (and generating) evidence, before final consensus. In its current form, the entire OMERACT instrument selection process must be repeated separately for RCTs, observational studies, and clinical practice. There is certainly a need for a coordinated approach to the assessment of $\mathrm{pSpA}$ in research and clinical practice settings to improve clinical integration and reporting of RCTs. The requirements of a measurement instrument may differ in each setting. For example, in the RCT setting, discrimination is a high priority whereas in observational research and routine clinical practice, feasibility may be a priority. In judging each of the instruments tested by Beckers et $\mathrm{al}^{5}$ through the OMERACT lens, there are encouraging results with respect to concurrent validity; however, each instrument has barriers that remain before wider adoption can be considered in the setting of $\mathrm{pSpA}$.

A final consideration when selecting, or developing, an instrument for the assessment of disease activity is the need to assess disease impact. Beckers et al discuss the discordance between instruments when classifying patients into the disease activity states. ${ }^{5}$ The discordance between patient and physician assessment of disease has been previously reported in a number of immune-mediated inflammatory diseases including PsA. ${ }^{9}$ In the study by Beckers et al, ${ }^{5}$ some of the discordance is likely to be due to the different components of the PASDAS, DAPSA, and ASDAS instruments. Each of these instruments has slightly different representations of patient and physician components and it is worth considering that impact should be assessed alongside activity, such that symptoms from noninflammatory causes can be addressed accordingly. Disease activity generally refers to reversible pathophysiological manifestations that can be ameliorated with effective pharmacological therapy. Impact of disease includes all the ways in which an individual can be affected by a disease, including irreversible manifestations such as damage and nondisease factors such as self-management. ${ }^{10}$ Disease activity and impact may require treatment escalation, but there are advantages in measuring disease activity and impact separately. GRAPPA voted that impact should be assessed separately from activity in PsA using the Psoriatic Arthritis Impact of Disease, ${ }^{11}$ and there may be advantages to a similar approach in the wider setting of $\mathrm{pSpA}$.

There is a clear, unmet need, particularly for patients with nonpsoriatic $\mathrm{pSpA}$, where interventional studies are very limited. The study by Beckers et $\mathrm{al}^{5}$ in this issue of The Journal provides information on some of the composite scores that could be utilized to measure treatment effect, alongside other measures previously studied in the ABILITY-2 population. However, the question of the gold standard in arthritis disease activity in 
the RCT and routine practice settings remains a complex issue. Future studies would benefit from both patient and physician anchor statements around disease activity and response to help identify the optimal tools for further research.

\section{REFERENCES}

1. Rudwaleit M, van der Heijde D, Landewe R, et al. The Assessment of SpondyloArthritis International Society classification criteria for peripheral spondyloarthritis and for spondyloarthritis in general. Ann Rheum Dis 2011;70:25-31.

2. Mease P, Sieper J, Van den Bosch F, Rahman P, Karunaratne PM, Pangan AL. Randomized controlled trial of adalimumab in patients with nonpsoriatic peripheral spondyloarthritis. Arthritis Rheumatol 2015;67:914-23.

3. Coates LC, Abraham S, Tillett W, et al. Performance and predictors of minimal disease activity response in peripheral spondyloarthritis patients treated with adalimumab. Arthritis Care Res 2020 Sep 16 (Epub ahead of print).

4. Turina MC, Ramiro S, Baeten DL, et al. A psychometric analysis of outcome measures in peripheral spondyloarthritis. Ann Rheum Dis 2016;75:1302-7.

5. Beckers E, Been M, Webers C, et al. Performance of 3 composite measures for disease activity in peripheral spondyloarthritis. J Rheumatol xxxxxxxxxxxx.
6. Taylor WJ, Harrison AA. Could the Bath Ankylosing Spondylitis Disease Activity Index (BASDAI) be a valid measure of disease activity in patients with psoriatic arthritis? Arthritis Rheum 2004;51:311-5.

7. Fernandez-Sueiro JL, Willisch A, Pertega-Diaz S, et al. Validity of the Bath Ankylosing Spondylitis Disease Activity Index for the evaluation of disease activity in axial psoriatic arthritis. Arthritis Care Res 2010;62:78-85.

8. Poddubnyy D. Identifier NCT04434885. Axial involvement in Psoriatic Arthritis Cohort (AXIS). [Internet. Accessed September 20, 2021.] Available from: https://clinicaltrials.gov/ct2/show/ NCT0 4434885

9. Sacristan JA, Dilla T, Diaz-Cerezo S, Gabas-Rivera C, Aceituno S, Lizan L. Patient-physician discrepancy in the perception of immune-mediated inflammatory diseases: rheumatoid arthritis, psoriatic arthritis and psoriasis. A qualitative systematic review of the literature. PLoS One 2020;15:e0234705.

10. Tillett W. Composite measures of impact and activity in psoriatic arthritis: a conceptual framework. J Rheumatol 2017;44:268-70.

11. Tillett W, McHugh N, Orbai AM, et al. Outcomes of the 2019 GRAPPA workshop on continuous composite indices for the assessment of psoriatic arthritis and membership-recommended next steps. J Rheumatol Suppl 2020;96:11-8. 